\title{
Asymmetric Spray and Multi-Forwarding for Delay Tolerant Networks
}

\author{
Yue Cao, Haitham Cruickshank and Zhili Sun \\ Centre for Communication System Research, \\ University of Surrey, Guildford, GU2 7XH, UK \\ \{Y.Cao,H.cruickshank,Z.Sun\}@Surrey.ac.uk
}

\begin{abstract}
The framework of Delay Tolerant Networks (DTNs) is recently received an extensive attention and its implementation aims ranging from Wireless Sensor Network to Interplanetary network. It has a promising future in military affairs, scientific research and exploration. With the characteristic of long delay, intermittent connectivity, limitation of buffer size and energy, the traditional routing algorithms in the Internet don't perform well for DTNs. In this paper, we propose an asymmetric spray mechanism combined with the concept of message classes called ASAMF. For each message class, a corresponding forwarding queue is designed and these asynchronous queues are scheduled according to their priorities. Compared with some state of art algorithms, our proposed algorithm achieves a better performance.
\end{abstract}

Keywords: Delay Tolerant Networks, Routing Algorithms

\section{Introduction}

The TCP/IP protocol has played an important role in the development of Internet because the IP protocol performs well to hide the heterogeneous properties of different subnetworks. Nevertheless, TCP/IP works under the assumptions such as end to end connectivity, relatively short round trip time (RTT) and low error rate. This is not possible for some challenged networks including wildlife tracking, Vehicle Ad hoc Networks (VANETs), interplanetary networks, military networks, pocket switched networks, underwater networks, free-space optical networks and rural Internet. Generally, these are intermittently connected because of the sparse infrastructure, non end-to-end connectivity and high mobility, thus are generally called challenge networks.

Delay Tolerant Networks (DTNs) [1] are designed to cope with these challenges. It makes use of scheduled, predicted and opportunistic connectivity and forms a store and forward overlay network to provide custody based message oriented transfer. Routing is the main challenge in DTNs since the characteristic of DTNs prevents the routing techniques in traditional networks from working effectively. Currently, many existing routing algorithms in DTNs have been proposed to enable message delivery in such challenge environment. Delivery ratio as the main performance objective is always taken into account. However, the performance of these algorithms creates more contention in terms of the connectivity and more energy consumption even if they can achieve a high delivery ratio. 
In general, the routing protocols must make a tradeoff between maximizing the message delivery ratio and minimizing resource consumption. On one hand, the ideal approach is to use the single copy approach for successful delivery. However on the other hand, the effective way to maximize the message delivery is to enlarge the number of message copies in the networks. Therefore, one feasible approach to reduce the overhead but maintain the high delivery ratio is to intelligently replicate and forward the messages.

The main contribution of this paper is to design an algorithm to achieve a high delivery ratio but with a low overhead as well as the relatively less latency and energy consumption. Our proposed algorithm mainly implements an asymmetric spray mechanism to boost the message dissemination to intermediate nodes that might be close to their destinations and enable these messages to be delivered before they are fully sprayed. Based on the characteristic of messages, we classify them into three classes and for each message class, a corresponding queue is proposed. In particular these corresponding queues are scheduled according to the priorities that are checked periodically. The energy function is also integrated since the low overhead ratio does not mean the low energy consumption.

In the following section, we briefly review the current taxonomy of unicast routing algorithms in DTNs, and then in section 3 we present our algorithm, which consists of six functions. Based on the simulation result in section 4 , we conclude our work the present the future direction in the last section.

\section{Related Work}

Excluding the assistance of additional infrastructure, the current taxonomy of unicast routing techniques in DTNs are classified into three main families which are single copy utility forwarding, multi copy naive replication and the hybrid families.

\subsection{Single Copy Utility Forwarding}

The algorithms in this family are perform based on the single copy model, which means the message carrier doesn't keep the copy of the forwarded message after the successful custody transfer.

The earlier link state based algorithms in [2] focus on the delay of each link state. Social networks as a newly research area proposed in the recent year utilizes the encounter relationship of each node [3]. Nevertheless, the single forwarding routing algorithms do not work efficiently in the sparse scenario or if the life time of message is quite small.

Other parameters such as energy, movement speed, node density and location can also be regarded as the consideration for routing decision. For example the Context Aware Routing (CAR) [4] utilizes the residual energy, dynamics of network topology and also integrates the synchronous and asynchronous mechanism. When the end to end connection is currently available then the system shifts to the traditional routing protocol to forward the message, also it uses the context information to decide the next hop when the continuous end to end connectivity is available. 


\subsection{Multi Copy Naive Replication}

The simplest algorithm is Direct Delivery [5], which only keeps the message until it encounters the destination. It is considered as a degraded naive replication based algorithm.

The Epidemic as the earliest multi-copy based algorithm is proposed in [6], each node doesn't implement the routing decision but just replicates the messages to neighbor nodes which are carrying this message. Provided that the buffer resource and bandwidth is large enough, Epidemic theoretically guarantees the shortest path for successful delivery. Nevertheless, the congestion due to the limited resource in reality is the main limitation of the scalability.

The Spray-and-Wait [7] combines the diffusion speed of Epidemic routing with the simplicity and thriftiness of Direct Delivery. For each message it defines an initial number of copy tickets to limit the number of replication and spreads them at each encounter opportunity to guarantee at least one of them can find the destination quickly, and then it stops and lets each node carrying the messages of which the copy ticket is one to perform direct delivery. Normally, the replication based routing algorithms can achieve a high delivery ratio but might obtain a high overhead ratio as well.

\subsection{Hybrid}

The algorithms in this family utilize the advantage of utility forwarding based routing algorithms and replication based algorithms, which could achieve a higher delivery ratio and relatively low overhead ratio in theory.

The Prophet [8] integrates the property of replication and prediction based forwarding. The sender always selects the neighbor nodes with the highest encounter probability and replicates its message to this candidate node. In addition, it also uses the transitivity to enhance the congestion avoidance.

The core concept of the MaxProp [9] protocol is a ranked list of the carried messages based on a cost for each destination. The cost is an estimate of visual end to end route failure possibility, initially the possibility for each pair of nodes is uniform distributed and updated according to the incremental averaging. Two thresholds are defined for calculation the dropping and forwarding priority of each message under the current buffer occupancy. In addition, MaxProp uses acknowledgments sent to all the nodes in the network to inform them to clear out the existing copies of the delivered messages.

The Spray-and-Focus [10] aims to optimize the Spray-and-Wait in the wait phase. Instead, it forwards the message to a potentially appropriate node according to the smaller timer value utility rather than just wait. Nevertheless, the performance is strongly affected by the specific mobility factor. 


\section{Our Designed ASaMF--Asymmetric Spray and Multi Forwarding}

The overall function flow chart of our proposed algorithm is illustrated in the Fig.1 and the specific functions are introduced in the following subsections.

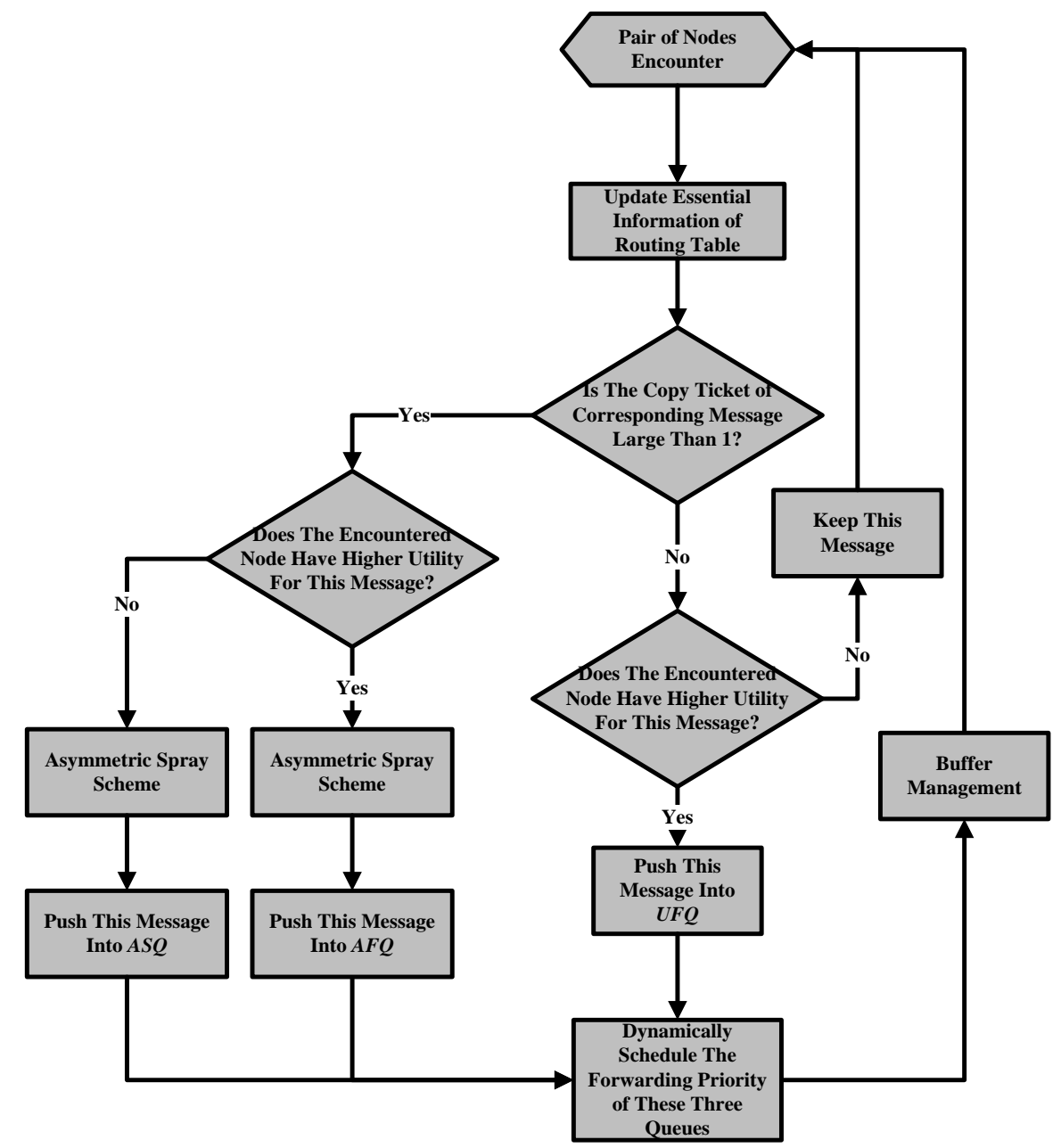

Fig. 1. Function Flow Chart of ASAMF

\subsection{Estimation Candidates' Utility}

Traditionally, the main problem in designing an efficient routing algorithm in DTNs is how to obtain the network topology information, due to the limited property of DTN node, it is difficult to obtain this global information. Some techniques in the 
related work assume that the partial history information can be used to anticipate the future encounter opportunity, however, it does not comprehensively take into account the mobility pattern. Assuming the future mobility pattern is known in advance is not reasonable in DTNs, herein our algorithm is designed based on these assumptions but makes use of the history network topology information.

Considering the mobility factor, we address the three conventional metrics, which are history encounter count, history encounter duration and history interval since pair of nodes encounter. In this paper, we use $N$ for history encounter count, $D$ for history encounter duration and $I$ for history encounter interval respectively.

We assume DTN node does not strictly move with a cyclic mobility pattern. To this end, we propose an iterative formula to smooth the effect of large variation within the number of encounters, where the value of local utility $(L U)$ is defined as:

$$
L U_{i, j}=\frac{\sum_{N_{i, j}=2}\left[\frac{D_{i, j}\left(N_{i, j}-1\right)}{I_{i, j}\left(N_{i, j}\right)}\right]}{\left(N_{i, j}-1\right)}
$$

where $i$ and $j$ are arbitrary pairwise encountered nodes. In detail, $N_{i, j}$ is the $N^{t h}$ encounter between $i$ and $j, D_{i, j\left(N_{i, j}-1\right)}$ is valid after the first encounter, with the time elapsing this property is useful because the node experiences a large number of encounters are more likely to successfully pass the message to the final destination than those who have infrequent encounters.

Normally, for each encounter opportunity the pairwise nodes would update their local routing information which contains a set of $L U$ for the nodes they encountered before. Nevertheless, there comes a challenge that estimating delivery potential of other nodes based on the local view is not reasonable since it might ignore the factor of its history encountered nodes.

We propose an approach to help the DTN node to reduce this limitation. For instance, when pairwise nodes encounter, firstly both of them would calculate and update their $L U$ on behalf of themselves. Afterwards, both of them would also add their local routing information to each other for the purpose of extending the knowledge. To this end, they can obtain the knowledge from their neighbors' history encounter information.

Based on the above analysis, a joint utility $J U$ is proposed, as in DTNs the transmission range of the device is assumed to be very limited and thus it is difficult to obtain and maintain the global network topology information by the broadcast mechanism:

$$
J U_{c, d}=\frac{\left(\sum_{k=1}^{n} L U_{c_{k}, d}\right)+L U_{c, d}}{\text { count }+1}
$$

where $n$ is the number of history encountered nodes of current carrier $c, c_{k}$ is the history encountered node of $c, d$ is the destination node.

In detail, count is initialized with zero and increased by one when $c_{k}$ contains the $L U$ for $d$. Therefore, the local node would obtain an abstract average knowledge for the destination not only based on itself but also based on the history information from its neighbors by Equation (2). As an example in Table 1, node $A$ encountered three nodes $X, Y, Z$, the $J U_{A, D 2}$ on behalf of $A$ is calculated as $\frac{0.2+0.5+0.6+0.3}{3+1}=0.4$. 
TABLE 1 EXAMPLE OF CALCULATION THE JU

\begin{tabular}{|c|c|c|c|c|}
\hline$L U_{A, D 1}$ & 0.2 & \multirow{3}{*}{ Encountered Node $X$} & $L U_{X, D 1}$ & 0.1 \\
\hline$L U_{A, D 2}$ & 0.3 & & $L U_{X, D 2}$ & 0.2 \\
\hline$L U_{A, D 3}$ & 0.1 & & $L U_{X, D 3}$ & 0.3 \\
\hline$L U_{A, D 4}$ & 0.5 & \multirow[t]{2}{*}{ Encountered Node $Y$} & $L U_{Y, D 1}$ & 0.4 \\
\hline$L U_{A, D 5}$ & 0.7 & & $L U_{Y, D 2}$ & 0.5 \\
\hline$L U_{A, D 6}$ & 0.8 & \multirow[t]{2}{*}{ Encountered Node $Z$} & $L U_{Z, D 2}$ & 0.6 \\
\hline$L U_{A, D 7}$ & 0.9 & & $L U_{Z, D 3}$ & 0.7 \\
\hline
\end{tabular}

\subsection{Asymmetric Spray}

Binary Spray-and-Wait as a classic algorithm has been proved and used in many scenarios because of its acceptable high delivery ratio and relatively low overhead ratio. However, it doesn't take into account the potential of candidate to deliver the messages to their destination, each node just naively sprays half numbers copy ticket of each message to any node encountered. Based on our joint uitiliy defined in the previous subsection, we assume that each node has a certain potential to forward the message to its destination at the initial time of the scenario. Therefore, on one hand, to equally spray the copy ticket might not be reasonable since to spray the half number of copy ticket to the encountered node with less $J U$ would waste some encounter opportunity. On the other hand, to unequally spray the copy ticket without any consideration is also unappropriate.

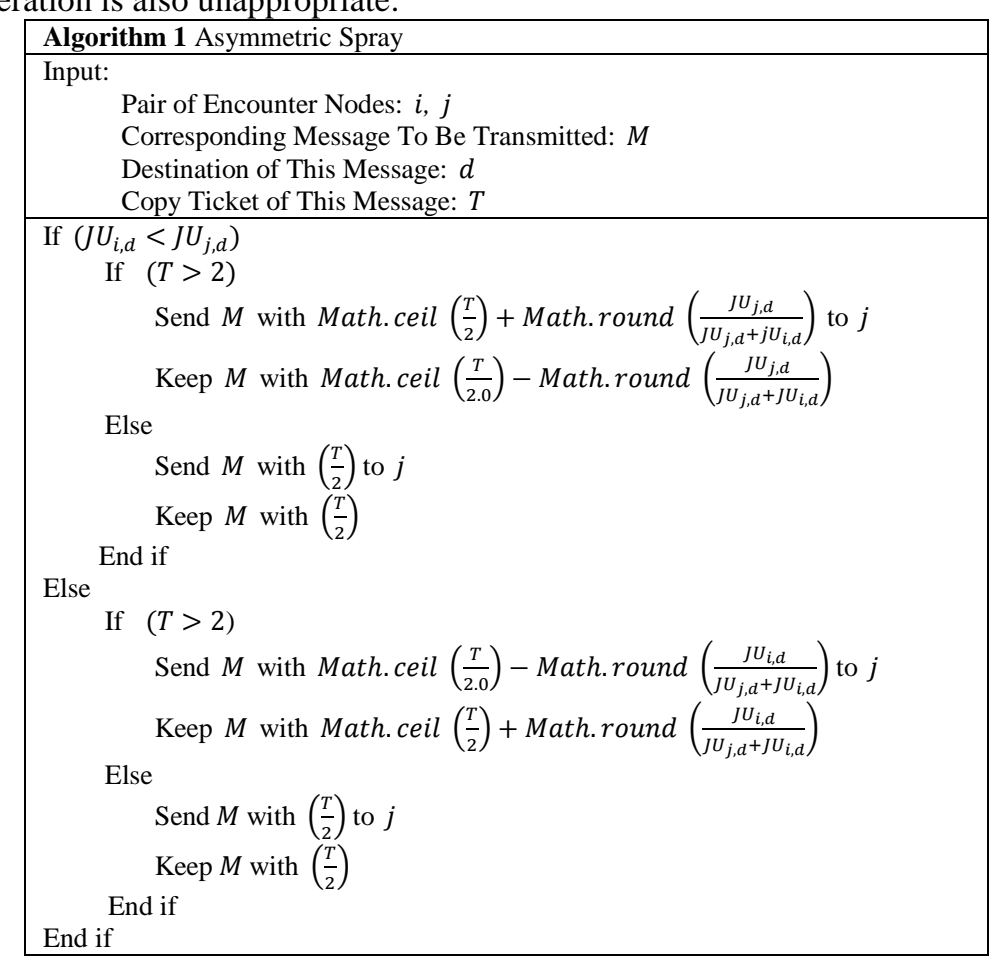


To this end, we propose a novel copy spray scheme based on binary Spray-andWait. For each message $M$ of which the destination is $d$ and with $T$ copy tickets in node $i$, When node $i$ has a lower $J U_{i, d}$ for this message's destination than $J U_{j, d}$ of encounterd node $j$. For this message $M$ that node $i$ occupies, node $i$ sprays more copy ticket $L_{j}$ of message $M$ to node $j$ and keeps less copy ticket $L_{i}$ by itself. In detail, we define the approximate delivery prediction $(D P)$ of the corresponding message in node $i$ before the spray process assumed it can be delivered before the message expiration:

$$
D P\left(J U_{i, d, T}\right)=1-\left(1-J U_{i, d}\right)^{T}
$$

After the spray process, the joint delivery prediction of node $i$ and $j$ are:

$$
D P\left(J U_{i, d, L_{i}} J U_{j, d, L_{j}}\right)=1-\left(1-J U_{i, d}\right)^{L_{i}} *\left(1-J U_{j, d}\right)^{L_{j}}
$$

where $T=L_{i}+L_{j}$, since $J U_{i, d}$ is smaller than $J U_{j, d}$, then:

$$
\begin{gathered}
D P\left(J U_{i, d, T}\right)=1-\left(1-J U_{i, d}\right)^{T}=1-\left(1-J U_{i, d}\right)^{L_{i}} *\left(1-J U_{i, d}\right)^{L_{j}} \\
\quad<1-\left(1-J U_{i, d}\right)^{L_{i}} *\left(1-J U_{j, d}\right)^{L_{j}}=D P\left(J U_{i, d, L_{i}} J U_{j, d, L_{j}}\right)
\end{gathered}
$$

Therefore, this spray mechanism can achieve a positive effect on maximization the message delivery ratio. Provided that $J U_{i, d}$ is larger than or equal to $J U_{j, d}$, inherently the best way is to keep the original copy ticket of this message until encounters a better candidate. Nevertheless, this behavior might result in the effect on the longer latency because the specific future prediction of mobility is independent of our assumption and we assume the poor candidate would encounter another better candidate in the future and then boosts the copy spray process. Therefore, we propose to spray less number of the copy ticket to the poor candidates.

\subsection{Multi-Forwarding Policies}

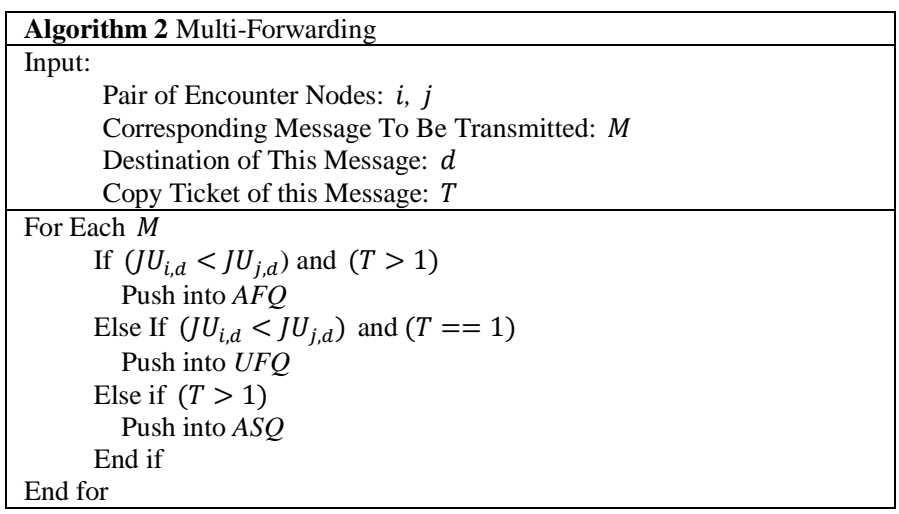

Algorithm 2 illustrates the routing decision process based on the message with different copy tickets.

\section{Asymmetric Forwarding Queue $(A F Q)$}

Upon the asymmetric spray proposed previously, for each currently available connection, node $i$ as the original sender will check whether the peer node $j$ on the other side of this connection has a larger $J U$ for the destination of the ongoing 
message $M$ to be sent. Particularly, we also check whether the message's copy ticket is larger than one. If this message accords with the above two conditions, then we push this message into Asymmetric Forwarding Queue $(A F Q)$. Basically, with the asymmetric spray mechanism, this message would be replicated to the candidate nodes that are close to this message's destination, hoping these candidates would spray the message to its destination faster.

Asymmetric Spray Queue (ASQ)

With respect to the encountered node with a smaller $J U$ for the destination than the current carrier, for each connection the current carrier would try its best to spray the copy ticket of all these messages in its buffer to the peer on the other side of the connection, which works as the mechanism of Spray-and-Wait but with asymmetric spray approach. The messages with more than one copy ticket under this mechanism are pushed into the Asymmetric Spray Queue $(A S Q)$.

\section{Utility Forwarding Queue (UFQ)}

Regarding the message of which the copy ticket is equal to one. This message cannot be sprayed but is performed as the utility based replication mechanism like Prophet. For each $M$ destined to its destination $d$ in node $i$, this message is replicated to the encountered node $j$ only if $J U_{i, d}<J U_{j, d}$. Accordingly, this message is pushed into the Utility Forwarding Queue $(U F Q)$. Otherwise it is kept by $i$ until it encounters a better candidate node.

\subsection{Message Priority Allocation}

The main motivation to define the message priority $(M P)$ is to forward the most appropriate message for each encounter opportunity. Totally different from the technique in traditional networks which focus on the message delay, herein we propose to address the delivery potential of each message. Based on the three forwarding queues proposed in the previous subsection, we classify the messages into multi copy based and single copy based, in addition, for messages in different queues, their priorities are defined respectively.

\section{Priority of Multi-Copy Based Message in $A F Q$}

The priority of message in this queue is defined as:

$$
(A F Q) M P=M U(e, d) * M C T
$$

where $e$ is the encountered node and $d$ is the destination of the message. In detail, $M C T$ is the message copy ticket and $M U$ is the message utility that is defined as $J U$, therefore these messages are scheduled according to the $J U$ of the encountered node. For example, if the encountered node has a higher $J U$ for the destination of $M_{1}$ than $M_{2}$, then $M_{1}$ is allocated with higher priority than $M_{2}$ if both of them are to be sent to this encountered node.

Regarding the messages of which copy tickets are larger than one, we try to forward them quickly to the intermediate nodes which might be close to the destinations of messages. Thus the message with higher $M U$ and larger MCT is forwarded prior to other messages.

Priority of Multi-Copy Based Message in $A S Q$ 
The main difference between the priority of messages in $A S Q$ and $A F Q$ is that the messages in this queue are scheduled based on the $J U$ of the current carrier and their copy ticket. It is defined as:

$$
(A S Q) M P=M U(c, d) * M C T
$$

where $c$ is the current carrier.

\section{Priority of Single-Copy Based Message in $U F Q$}

For messages that are processed as this taxonomy, their priorities are defined as:

$$
(U F Q) M P=\frac{M U(e, d)}{\text { Message } T T L}
$$

If the message with higher $M U$ but with a very limited remaining life time, it is regarded to be a high emergent message To this end, as the priority proposed, the message that has high potential to be delivered to its destination and low remain life time is always guaranteed to exist in the networks, which plays a positive effect on maximizing the delivery ratio.

\subsection{Forwarding Policies Scheduling}

Inherently, the queues for multi-copy messages should be scheduled prior to the queue for message with single copy. The main reason is that for messages which are processed in asymmetric forwarding queue and asymmetric spray queue, these replicated messages are allocated with the dedicated copy ticket. If their life time expires, the worst case is the messages with maximum copy ticket are cleared out from the buffer space. Under this case, it might reduce the delivery ratio to a largest extent. This analysis also accords with our message priority defined in the previous subsection.

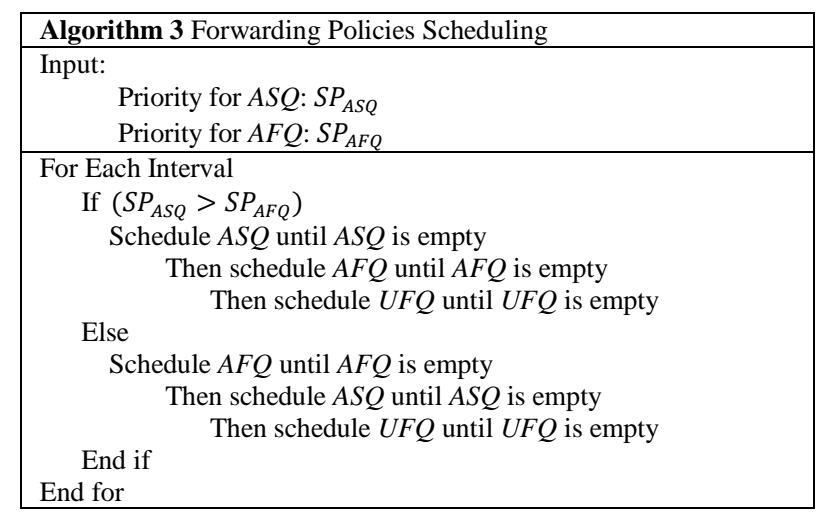

Nevertheless, it brings up a question that how to schedule the priority of asymmetric forwarding queue and asymmetric spray queue. For this purpose, we define a metric called scheduling priority $(S P)$ for these two queues.

$$
S P=\frac{\sum_{k=1}^{m}[M U(c, d) * M C T]}{m}
$$

where $m$ is the number of messages in the queue. For each interval, the node will check the current $S P$ of these two queues. To this end, as proposed in the DTNs RFC, we classify the messages into three classes which are bulk, normal and expedite, in 
the meanwhile they are processed according to the specific forwarding policies and scheduled according to their priorities respectively.

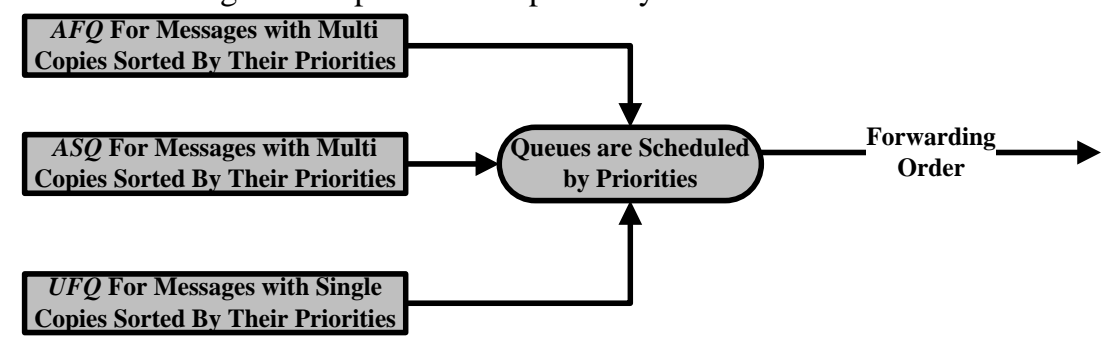

Fig. 2. Forwarding Queue Policy

\subsection{Buffer Management}

Normally, the storage is also limited in the restricted scenario and accordingly a node may not be able to deliver all of the messages. Hence a reasonable buffer management function is essential.

We still utilize the message priority mentioned in the previous subsection. Herein, we define the drop priority $(D P)$ of the message as:

$$
D P=M U(c, d) * M C T
$$

We classify the messages into multi copy based and single copy based, and they are pushed into different bins respectively. In detail, for messages with single copy, MCT is equal to one. Normally the messages in the bin for multi copy messages are firstly discarded from the lowest priority. If there are no more messages in this bin, then the messages in the single copy bin are discarded from the lowest priority.

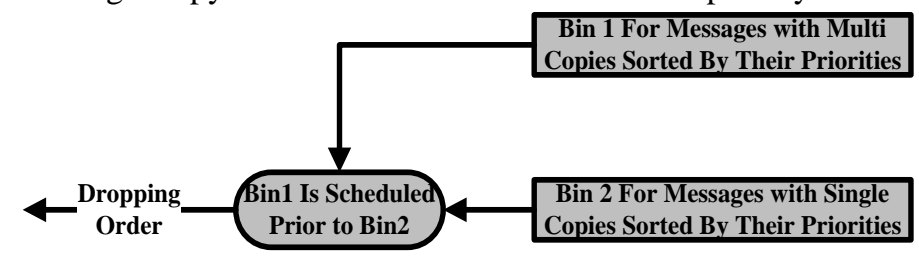

Fig. 3. Drop Policy

Consideration behind this approach is that the message with lowest copy ticket and lowest delivery prediction for its destination would be more useless since most of its copy has been sprayed to the intermediate nodes that are close to its destination. The messages with one copy ticket are assumed to be close to the destination and therefore they are discarded with lowest priority once there are no more messages with multi copy ticket.

To ease such redundant transmissions, the destination will generate an acknowledgement of which the size can be ignored compared to the size of messages when it successfully receives this message, and this acknowledgement will be flooded to the entire network. Intermediate nodes receive this acknowledgement will check their buffer and discards the message which has been successfully received. 


\section{Simulation Results}

TABLE 2 SIMULATION CONFIGURATIONS

\begin{tabular}{|l|l|}
\hline Simulation Time & $43200 \mathrm{~s} \quad$ (12 hours) \\
\hline \multirow{2}{*}{ Connectivity (Bluetooth) } & Bandwidth: $2 \mathrm{Mb} / \mathrm{s}$ \\
& Transmission Range: $10 \mathrm{~m}$ \\
\hline \multirow{3}{*}{ Message } & Generating Interval: $30 \mathrm{~s}$ \\
& Size: 200kB $-2 \mathrm{MB}$ \\
& Initial TTL: 240 minutes \\
\hline Buffer Size & $10 \mathrm{MB}$ \\
\hline Energy per node & Initial Energy: $850 \mathrm{~mA} / \mathrm{h}$ \\
& Scanning Energy:38.61mA/h \\
& Transmission Energy: $51.47 \mathrm{~mA} / \mathrm{h}$ \\
\hline Number of Nodes & 126 \\
\hline Interval for Scheduling Model & $0.1 \mathrm{~s}$ \\
\hline Mobility & Helsinki Scenario \\
\hline
\end{tabular}

The simulation is implemented by Opportunistic Network Environment (ONE) [11] simulation environment. We evaluate the Spray-and-Focus (SaF), binary Spray-andWait (SaW), Epidemic, Prophet and MaxProp for comparison. Energy function is also integrated into all these algorithms. For the purpose of fairness, the initial number of copies for $\mathrm{SaF}, \mathrm{SaW}$ is set to 13, which is a recommended value between 10 and $15 \%$ nodes in the scenario. The definition of the main performance metrics are:

$$
\begin{gathered}
\text { Delivery Ratio }=\frac{\text { Total Delivered Messages }}{\text { Total Generated Messages }} \\
\text { Overhead Ratio }=\frac{\text { Total Relayed Messages-Total Delivered Messages }}{\text { Total Generated Messages }} \\
\text { Average Latency }=\frac{\text { Sum of Latency of Delivered Messages }}{\text { Total Generated Messages }}
\end{gathered}
$$

\subsection{Effect of Buffer Size}

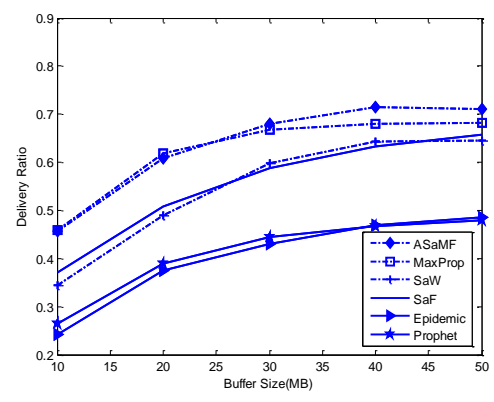

Fig. 4. Delivery Ratio vs Buffer Size

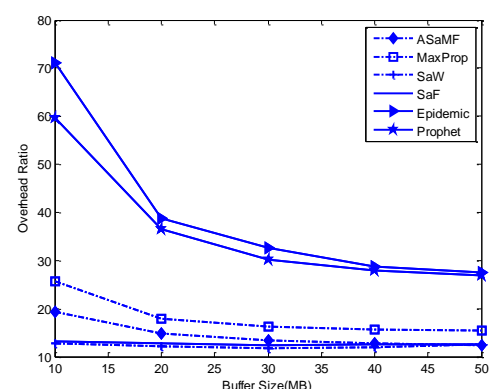

Fig. 5. Overhead Ratio vs Buffer Size 
In Fig.4, both Epidemic and Prophet achieve the lowest delivery ratio because of their naive replication based characteristics. $\mathrm{SaW}$ and $\mathrm{SaF}$ limit the initial copy ticket of messages so they can keep the messages in the buffer space to the maximum extent and forward them to encountered peers. Compared with MaxProp, which is regarded as a preeminent one for comparison, our algorithm achieves higher delivery ratio particular when the buffer size increases.

With respect to the overhead ratio in Fig.5, our ASaMF achieves the nearly smaller overhead ratio as the value of $\mathrm{SaW}$ and $\mathrm{SaF}$. As we reviewed, $\mathrm{SaF}$ uses more encounter opportunity during the focus phase whereas $\mathrm{SaW}$ just implements Direct Delivery in its second phase, this results in a higher overhead ratio of SaF compared with SaW. Even if MaxProp is designed with the dedicated buffer management, our proposed algorithm still outperforms MaxProp.

In Fig.6, our proposed algorithm also achieve the lowest average latency among other algorithms. Particularly, as we discuss in previous section, our asymmetric spray mechanism partially plays the important role on this good performance. Another contribution comes from the message priority, which aims to forward the most appropriate message for the current available connection.

Energy issue as a new consideration is taken into account in DTN routing recently. According to our result in Fig.7, though ASaMF and SaW achieves the nearly same overhead ratio. Inherently, our algorithm performs a replication based mechanism when the message copy ticket is equal to one, which occupies more buffer space and might abort some message due to the mobility factor. Therefore, it requires the retransmission of the messages which have been aborted. For this reason, it comsumes more energy than $\mathrm{SaW}$ and $\mathrm{SaF}$.

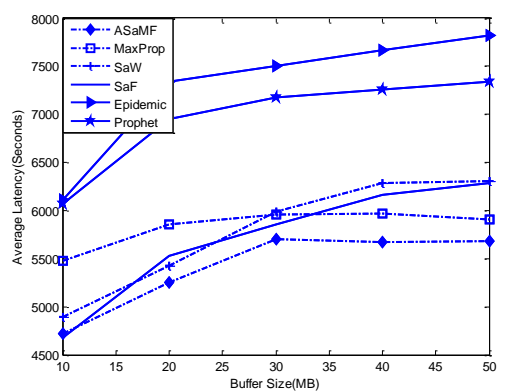

Fig. 6. Average Latency vs Buffer Size

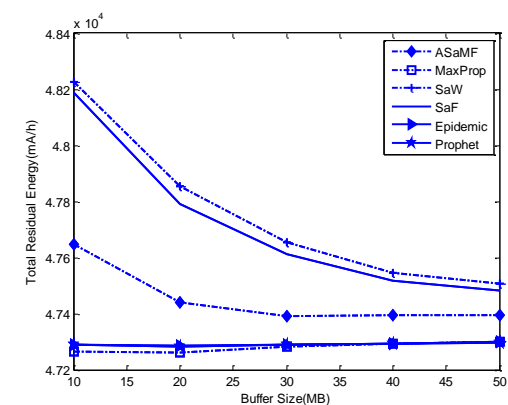

Fig. 7. Total Residual Energy vs Buffer Size

\subsection{Effect of Message TTL}

In this section, we fix the buffer size as $50 \mathrm{MB}$ but vary the value of message TTL.

When the message TTL increases in Fig.8, our algorithm still outperforms other algorithms. MaxProp with a dedicated buffer management also performs well compared with $\mathrm{SaF}$ and $\mathrm{SaW}$, which are not designed with any buffer management function. Due to the limited resource, the performance of Epidemic and Prophet degrades in terms of the larger message TTL.

The inherent charactersitic of our algorithm determines its overhead would be little higher than SaW and SaF in Fig.9. However the difference is close when the message 
TTL increased, this is because the asymmetric spray mechniasm work effectively since it sprays the message to the corresponding candidate nodes before its expiration .

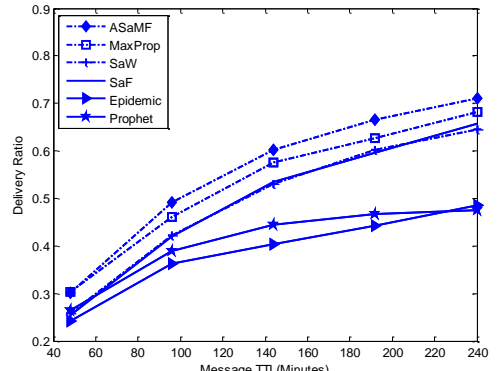

Fig. 8. Delivery Ratio vs Message TTL

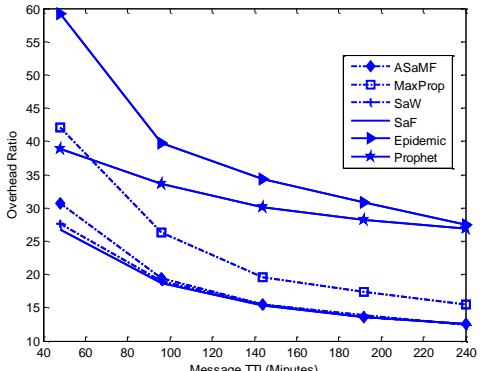

Fig. 9. Overhead Ratio vs Message TTL

In Fig.10, with respect to the average latency, our algorithm achieves the lowest latency which is similar to the result affected by the buffer size.

Due to the large message TTL, the messages in the buffer might be dropped particularly under the implementation the replication based algorithms. thus the current carrier would require more messages which have been cleared from the its buffer, this results in a more energy consumption. According to the result in the Fig.11, based on the the overall performance, $\mathrm{SaW}$ and SaF saves more energy due to the less number of transmission. Relatively, Prophet does not achieve a acceptable delivery ratio even if it consumes the least energy. Our algorithm balances the energy consumption and the deliver ratio, thus it is energy efficiency.

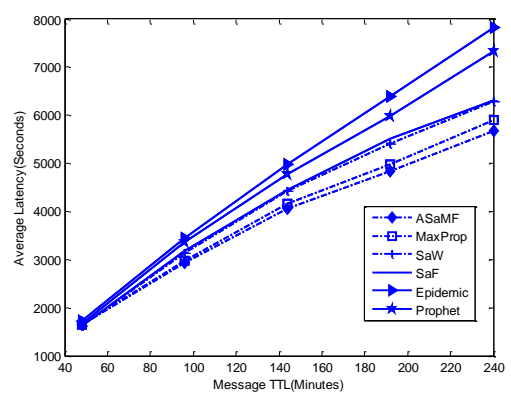

Fig. 10. Average Latency vs Message TTL

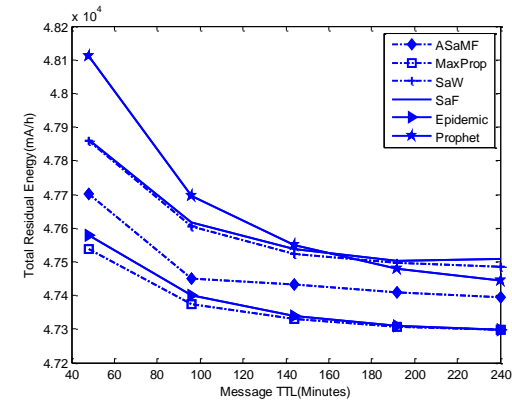

Fig. 11. Total Residual Energy vs Message TTL

\section{Conclusion and Future Work}

The ability to efficiently forward a message and appropriately select the route through intermittently connected networks is critically important in DTNs. As we review in our paper, many utility based replication algorithms in hybrid family can achieve relatively high delivery ratio but still obtains a higher overhead ratio. Besides, limited buffer space would reduce the performance due to the congestion as well as energy constraint in the devices. With a novel multi-forwarding model based on dynamic message classification and an asymmetric spray scheme, our proposed algorithm 
comprehensively outperforms other existing state of art algorithms in terms of message delivery ratio, overhead ratio and average latency with lower energy consumption as well. With respect to our future work, we plan to further analyze linear characteristic of the asymmetric spray to achieve the delivery ratio to the maximum extent and lower overhead ratio as well, and focus on the asymmetric spray we will further focus on the route decision to reduce the number of relay. Finally, the issue of message classes is still in infancy and we will also address our dynamic classification mechanism combined with the QoS service requirement as well.

\section{References}

1. Fall, K., Farrell, S.: DTN: an Architectural Retrospective", IEEE Journal on Selected Areas in Commun. vol.26, no.5, pp.828-836, June (2008)

2. Jain, S, Fall, K and Patra R.: Routing in a Delay Tolerant Network. In: SIGCOMM 2004. Portland, September (2004)

3. Daly, E., Haahr M.: Social Network Analysis for Routing in Disconnected DelayTolerant MANETs. In Proc of the 8th ACM international symposium on Mobile ad hoc networking and computing. Montréal, Québec, Canada (2007)

4. Musolesi, M., Masclo, C.: CAR: Context Aware Adaptive Routing for Delay Tolerant Mobile Networks. IEEE Transaction on Mobile computing, (2009)

5. Grossglauser M., Tse DNC. Mobility increases the capacity of ad hoc wireless networks. IEEE/ACM Trans. on Networking, (2002),10(4):477-486.

6. Becker, D.: Epidemic routing for partially connected ad hoc networks. Technique Report, CS-2000-06, Department of Computer Science, Duke University, Durham, NC, (2000)

7. Spyropoulos, T., Psounis, K., Raghavendra, C. Spray and Wait: an Efficient Routing Scheme for Intermittently Connected Mobile Networks. In: ACM 2005. USA, August (2005)

8. Lindgren A., Doria A., Schelén O.: Probabilistic Routing in Intermittently Connected Networks. In Proc. SIGMOBILE Mobile Computing Communications Review. July (2003)

9. Burgess, J., Gallagher, B., Jensen, D.: MaxProp: Routig for Vehicle-Based Disruption Tolerant Networking. In: IEEE INFOCOM 2006. Barcelona.April (2006)

10.Spyropoulos T., Psounis, K., Raghavendra, C.: Spray and Focus: Efficient mobility Assisted routing for Heterogeneous and Correlated Mobility. In: Fifth Annual IEEE International conference on Pervasive Computing and Communications Workshops. Newyork.March (2007)

11.Keränen, A., Ott, J., Kärkkäinen, T.: The ONE Simulator for DTN Algorithm Evaluation.In: SIMUTools'09: 2nd International Conference on Simulation Tools and Techniques. Rome, March (2009) 